\title{
Studies of Diboson Production and Triple Gauge Coupling at LHC
}

Zhengguo Zhao

University of Michigan, Ann Arbor, USA

On behalf of ATLAS and CMS

ICHEP06, 7/26-8/2, Moscow, Russia 


\section{Outline}

- Introduction

- Diboson production at LHC

- Triple gauge boson couplings

- Studies with full simulation data

- WZ, ZZ from CMS

- WZ, WW and ZZ from ATLAS

- Summary 


\section{Diboson Production at Hadron Machine}

$q$

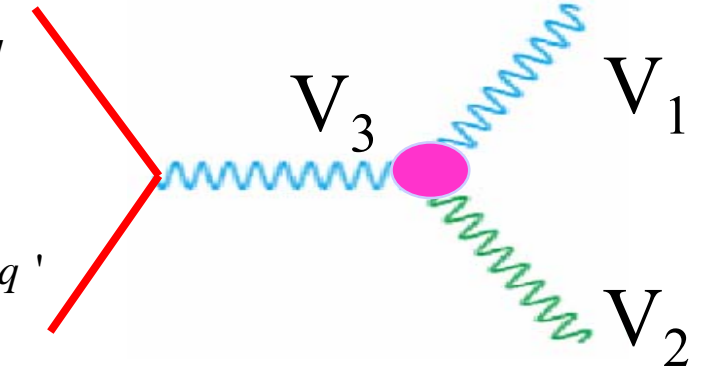

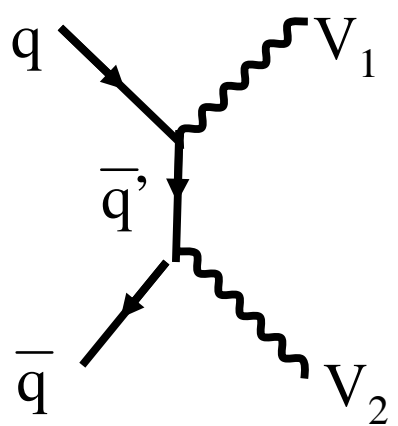

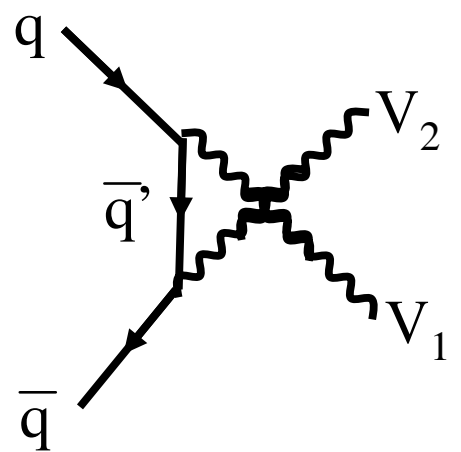

- LO Feynman diagram, $V_{1}, V_{2}, V_{3}=Z, W, \gamma \rightarrow W W, Z W, Z Z, W \gamma$.

- Only s channel has three boson vertex

- Diboson final states have predictable $\sigma_{\text {production }}$ and manifest the gauge boson coupling

SM:

- Pure neutral vertexes $Z Z Z Z, Z Z \gamma$ are forbidden ( $Z / \gamma$ carry no charge and weak isospin that needed for gauge bosons couple)

- Only charged couplings WW $\gamma$, WWZ are allowed 


\section{Study of WZ, WW and ZZ at LHC}

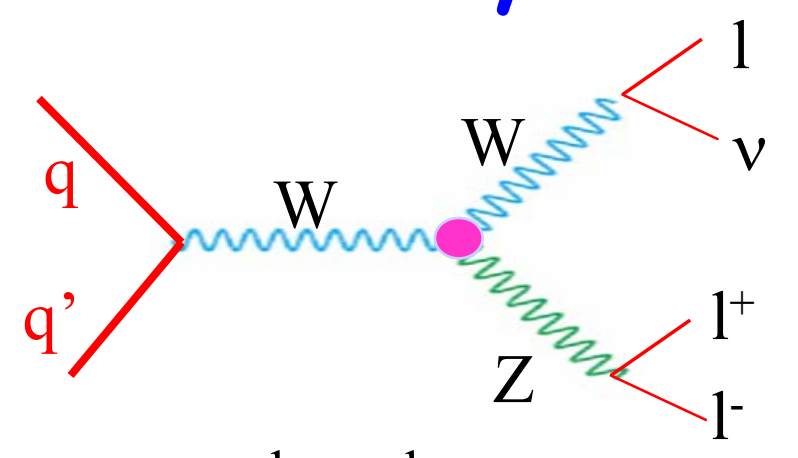

- s-channel dominates, $\sigma(S M)=57.7 \mathrm{pb}$

- Sensitive only to WWZ coupling

- Clean signal eee, ee $\mu, \mu \mu e, \mu \mu \mu$

- 3 isolated high $p_{T}$ leptons with large $E_{T}$ (miss)

s-channel

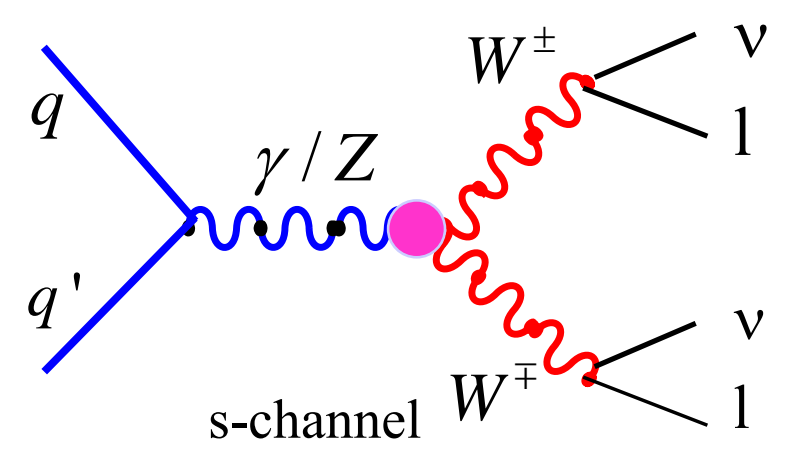

- $\sigma(S M)=127.5 \mathrm{pb}$

- Sensitive to WWZ and WW

- Clean signal ee, $\mu \mu$, e $\mu$

- 2 isolated high $p_{T}$ leptons with opposite charge and large missing $E_{T}$

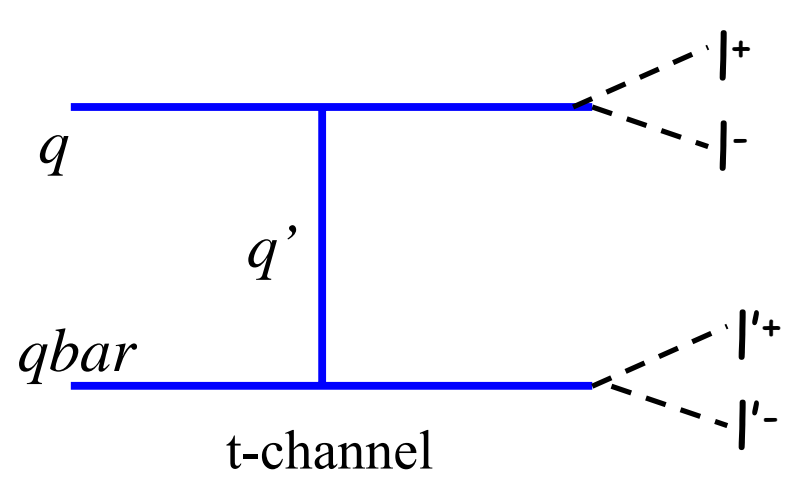

- s channel suppressed by $O\left(10^{-4}\right)$

- Only t-channel at tree level, $\sigma(S M)=16.8 \mathrm{pb}$

- 4 isolated high $p_{T}$ leptons from the $Z$ pair decays

- Clean signal eeee, ее $\mu \mu, \mu \mu \mu \mu$, almost bkg free 


\section{Triple Gauge Boson Couplings}

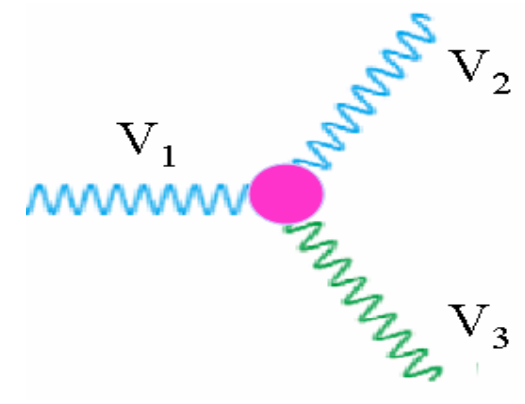

$$
\begin{aligned}
& L_{W W} / g_{W W}=i g_{1}^{V}\left(W_{\mu \nu}^{+} W^{\mu} V^{\nu}-W_{\mu}^{+} V_{v} W^{\mu v}\right) \\
& +i \kappa_{V} W_{\mu}^{+} W_{v} V^{\mu v}+i \frac{\lambda_{V}}{M_{W}^{2}} W_{\lambda \mu}^{+} W_{v}^{\mu} V^{\nu \lambda}
\end{aligned}
$$

- Characterized by an effective

$$
\begin{aligned}
& -g_{4}^{V} W_{\mu}^{+} W_{v}\left(\partial^{\mu} V^{v}+\partial^{\prime} V^{\mu}\right) \\
& +g_{5}^{V} \varepsilon^{\mu \nu \rho \alpha}\left(W_{\mu \nu}^{+} \partial_{\rho} W_{v}\right) V_{\alpha} \\
& +i \tilde{\kappa}_{V} W_{\mu}^{+} W_{v} \tilde{V}^{\mu v}+i \frac{i \tilde{\nu}_{v}}{M_{W}^{2}} W_{\mu \mu}^{+} W_{v}^{\mu} \tilde{V}^{\mu \nu}
\end{aligned}
$$

Lagrangian, parameterized in

terms of coupling parameters

for new physics

- $C, P$ and $C P$ symmetry conservation, 5 free parameters:

$-\lambda_{y}, \lambda_{z}:$ grow with $s$, big advantage for LHC

$-\Delta \kappa_{\gamma}=\kappa_{\gamma}-1, \Delta \mathrm{g}_{1} \mathrm{Z}=\mathrm{g}_{1} \mathrm{Z}_{-} 1, \Delta \kappa_{\mathrm{Z}}=\kappa_{\mathrm{Z}}-1$ : grow with $\sqrt{\mathrm{s}}$

- Tree level SM: $\lambda_{\gamma}=\lambda_{\mathrm{Z}}=\Delta \kappa_{\gamma}=\Delta \mathrm{g}_{1}{ }^{\mathrm{Z}}=\Delta \kappa_{\mathrm{Z}}=0$ 


\section{Anomalous Coupling and Form Factor}

- Cross section increase for coupling with non-SM values, yielding large cross section at high energies that violating tree level unitarity $\rightarrow$ form factor scale

$$
a(s)=\frac{a_{0}}{\left(1+s / \Lambda_{F F}^{2}\right)^{2}}
$$

s: subprocess CM energy. $\Lambda$ : form factor scale

- TGCS manifest in

- cross section enhancement

- high $\mathrm{p}_{\mathrm{T}}(\mathrm{V}=\mathrm{Z}, \mathrm{W}, \gamma)$

- production angle 


\section{LHC Expectations for the TGCS}

LHC

- High CM energy $\rightarrow$ larger $\sigma$

- High luminosity $\rightarrow$ high statistics

- High sensitivity

$\rightarrow$ Expected to be $\sim \times 10$ improvement on LEP/Tevatron

Predictions for TGCs at $95 \%$ C.L. for $L=30 \mathrm{fb}^{-1}$ (inc syst)

$$
\begin{gathered}
-0.0035<\lambda_{\gamma}<+0.0035 \\
-0.0073<\lambda_{\mathrm{Z}}<+0.0073 \\
-0.075<\Delta \kappa_{\gamma}<+0.076 \\
-0.11<\Delta \kappa_{\mathrm{Z}}<+0.12 \\
-0 . .86<\Delta \mathrm{g}_{\mathrm{Z}}^{1}<+0.011
\end{gathered}
$$




\section{Motivations}

- Measure dibson production $\sigma$ and TGCS

- Explore none-Abelian $S U(2) \times U(1)$ gauge structure of $S M$ and test the central part of the SM

- Probe new physics if production cross section, or TGCS deviate from SM prediction $\rightarrow$ complementary to direct search for new physics

- Understand the backgrounds of many important physics analyses

Search for Higgs, SUSY, graviton and study of ttbar 


\section{Diboson as Background}
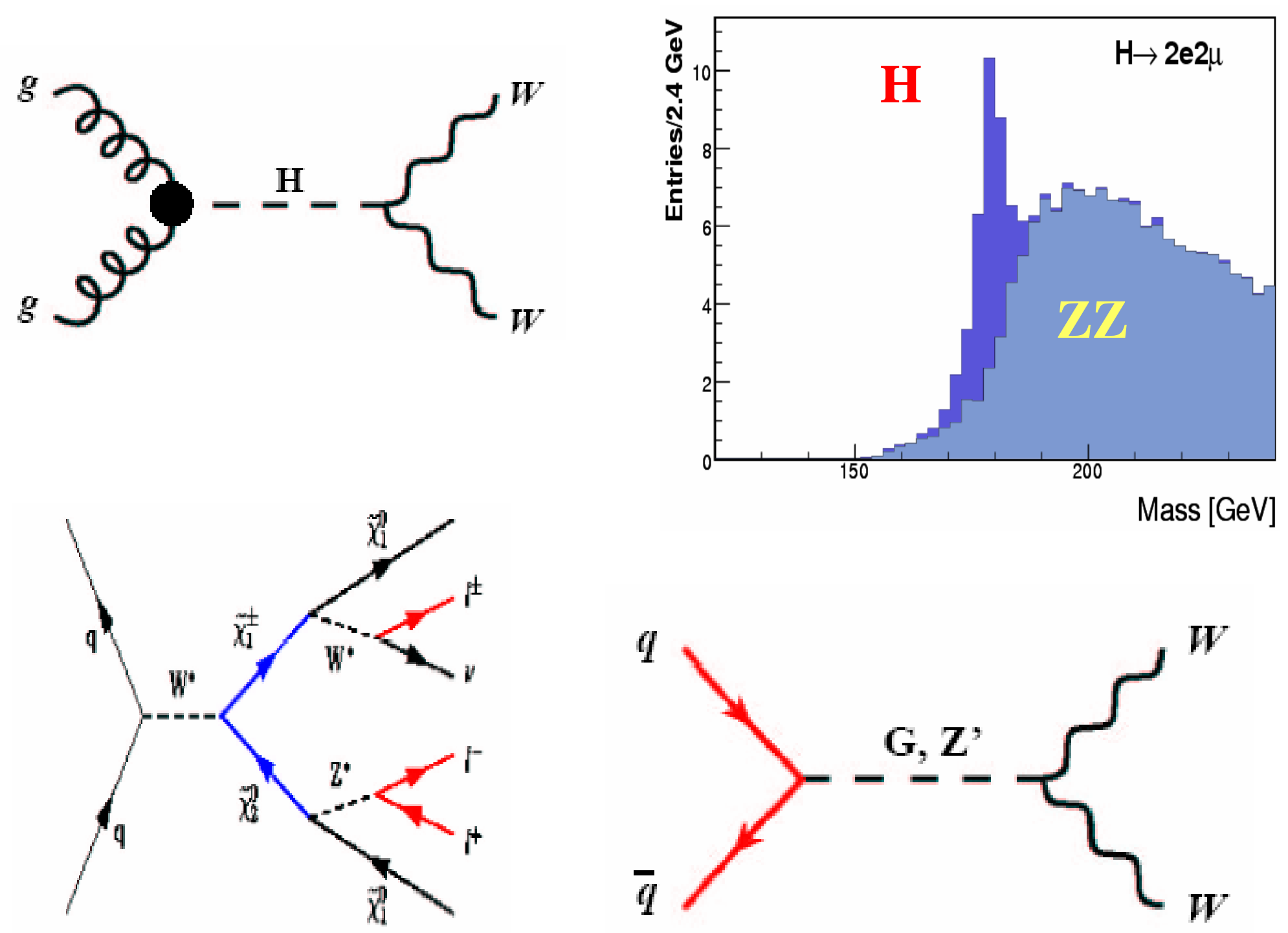


\section{Signal and Background Samples (CMS)}

Signal $\left\{\begin{array}{|c|c|c|}\hline & \sigma \times B r & \text { k-factor } \\ \hline \mathrm{Z} Z \mathrm{ZZ}(4 \mathrm{e}) & 18.7 \mathrm{fb} & 1.3 \\ \hline \mathrm{WZ}(3 \mathrm{I}, \mathrm{I}=e, \mu, \tau) & 1.6 \mathrm{pb} & 1.92 \\ \hline \mathrm{ttbar}(2 \mathrm{l}) & 62.3 \mathrm{pb} & 1.6 \\ \hline \mathrm{Z}(e e) \mathrm{bb} & 60.3 \mathrm{pb} & 2.4 \\ \hline \mathrm{Z}(\mu \mu) \mathrm{bb} & 60.3 \mathrm{pb} & 2.4 \\ \hline \mathrm{ttbar}(4 e) & 194 \mathrm{fb} & 1.6 \\ \hline \mathrm{ZZ}(2 e 2 \mu) & 32.3 \mathrm{fb} & 1.35 \\ \hline\end{array}\right.$

t+(2l) generated with TopReX, Zbb with CompHEP, all others with Pythia 


\section{WZ $\rightarrow 3 \mid$ Expected Signal \& Background}

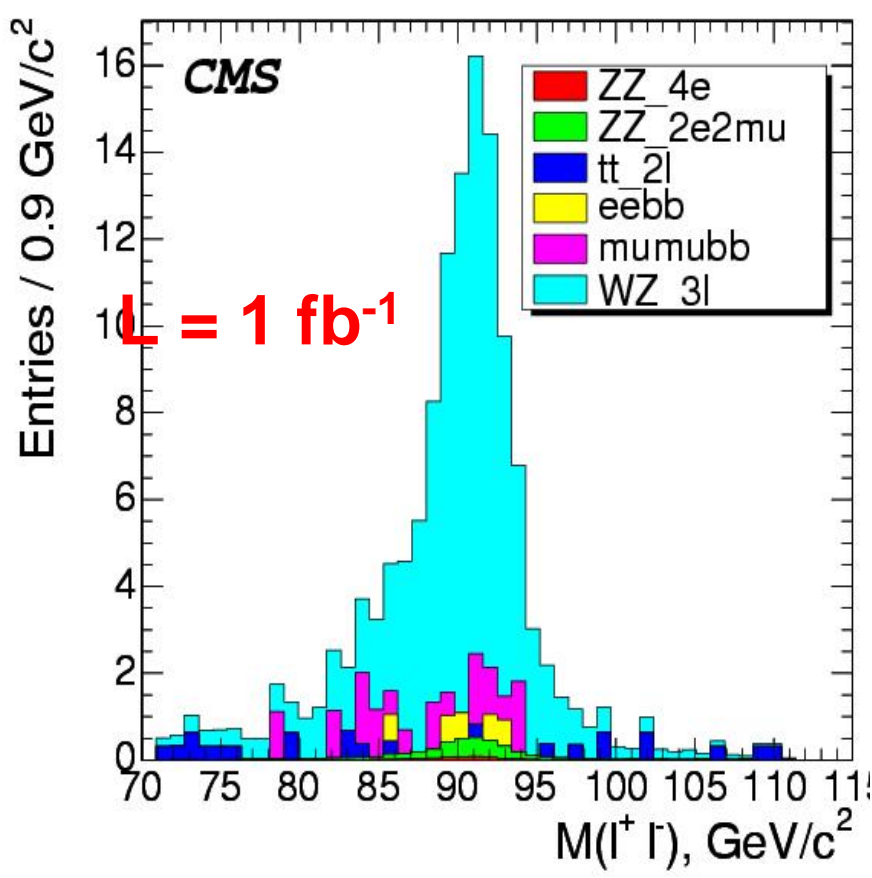

- $\left.M\left(I^{+}\right)^{-}\right)$after all cuts 4 channels combined $(3 e, 2 e 1 \mu, 2 \mu 1 e, 3 \mu)$

- Presence of peaking backgrounds

- Zbb

- ZZ (irreducible)

- High significance in the first $1 \mathrm{fb}^{-1}$

Expected signal and background yields for $1 \mathrm{fb}^{-1}$

\begin{tabular}{lcccccl}
\hline \hline & $e^{ \pm} e^{+} e^{-}$ & $\mu^{ \pm} e^{+} e^{-}$ & $e^{ \pm} \mu^{+} \mu^{-}$ & $\mu^{ \pm} \mu^{+} \mu^{-}$ & Total & Efficiency, \% \\
\hline$W^{ \pm} Z^{0} \rightarrow \ell^{ \pm} \ell^{+} \ell^{-}$ & 14.8 & 26.9 & 28.1 & 27.0 & 96.8 & 6.1 \\
\hline$Z^{0} Z^{0}$ & 0.63 & 1.54 & 1.50 & 1.51 & 5.19 & 4.7 \\
$t \bar{t}$ & 0.93 & 1.55 & - & 0.31 & 2.79 & 0.02 \\
$\mu^{+} \mu^{-} b \bar{b}$ & - & - & 6.54 & 4.9 & 11.4 & 0.005 \\
$e^{+} e^{-} b \bar{b}$ & 1.21 & 1.82 & - & - & 3.03 & 0.005 \\
\hline Total background & 2.8 & 4.9 & 8.0 & 6.7 & $(22.5)$ & - \\
\hline$S_{L}$ & 5.3 & 7.3 & 6.5 & 6.6 & 12.8 & - \\
\hline \hline
\end{tabular}




\section{$\mathrm{ZZ} \rightarrow 4 e$ Expected Signal \& Background}

$M\left(e^{+} e^{-}\right)$after all cuts

( 2 entries per event)

Nearly background free!

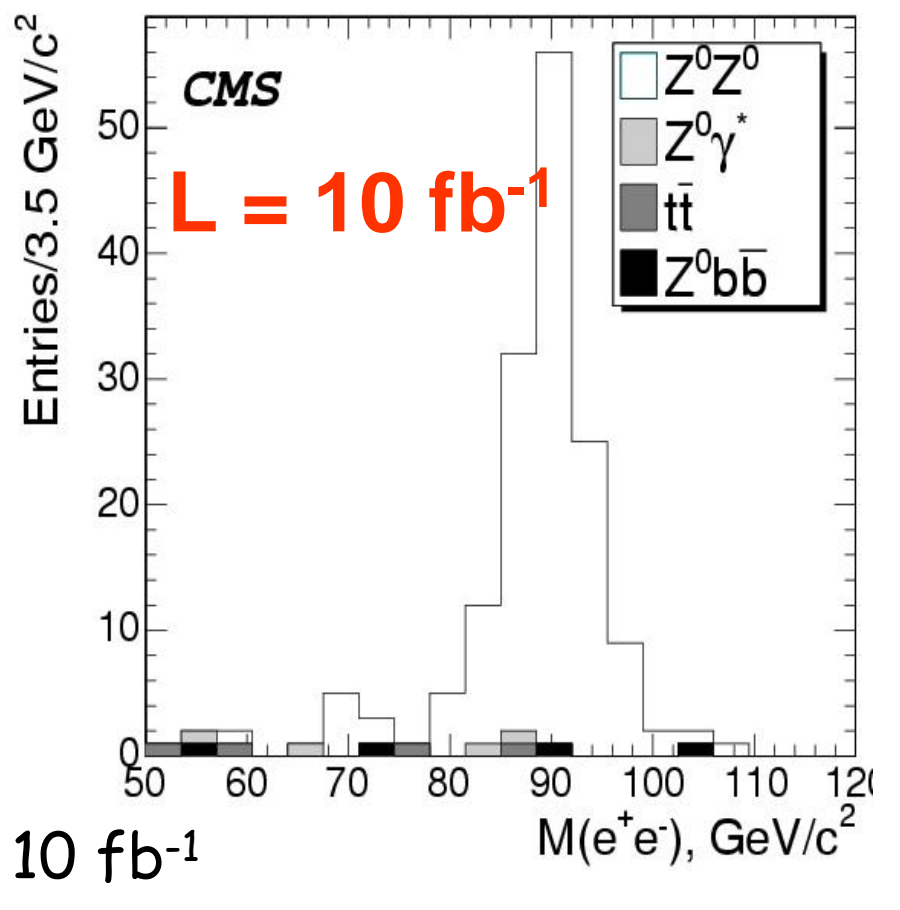

Expected signal and background yields for 1 and $10 \mathrm{fb}^{-1}$

\begin{tabular}{lccc}
\hline \hline & Efficiency, \% & $N_{\text {events }} / 1 \mathrm{fb}^{-1}$ & $N_{\text {events }} / 10 \mathrm{fb}^{-1}$ \\
\hline$Z^{0} Z^{0}$ & 38 & $(7.1)$ & 71.1 \\
\hline$Z^{0} \gamma^{*}$ & 4.5 & 0.16 & 1.60 \\
$Z^{0} b \bar{b}$ & 0.07 & 0.08 & 0.84 \\
$t \bar{t}$ & 0.06 & 0.12 & 1.22 \\
\hline Total background & - & 0.36 & 3.66 \\
\hline$S_{L}$ & - & 4.8 & 13.1 \\
\hline \hline
\end{tabular}




\section{WZ and ZZ Discovery Potential (CMS)}

\section{$Z Z \rightarrow 4$ e signal significance}

$W Z \rightarrow 3$ I signal significance

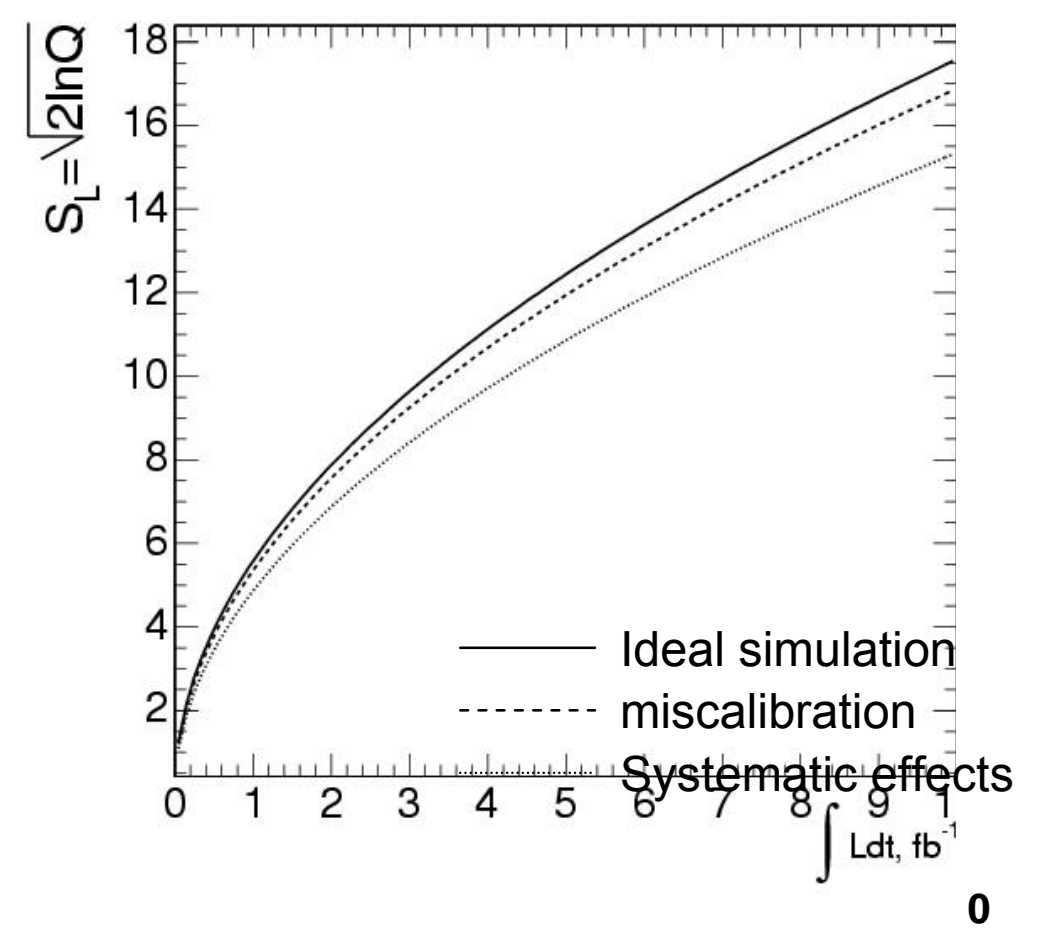

$S_{L}=\sqrt{2 \ln Q}, \quad Q=\left(1+\frac{N_{S}}{N_{B}}\right)^{N_{S}+N_{B}} e^{-N_{S}}$

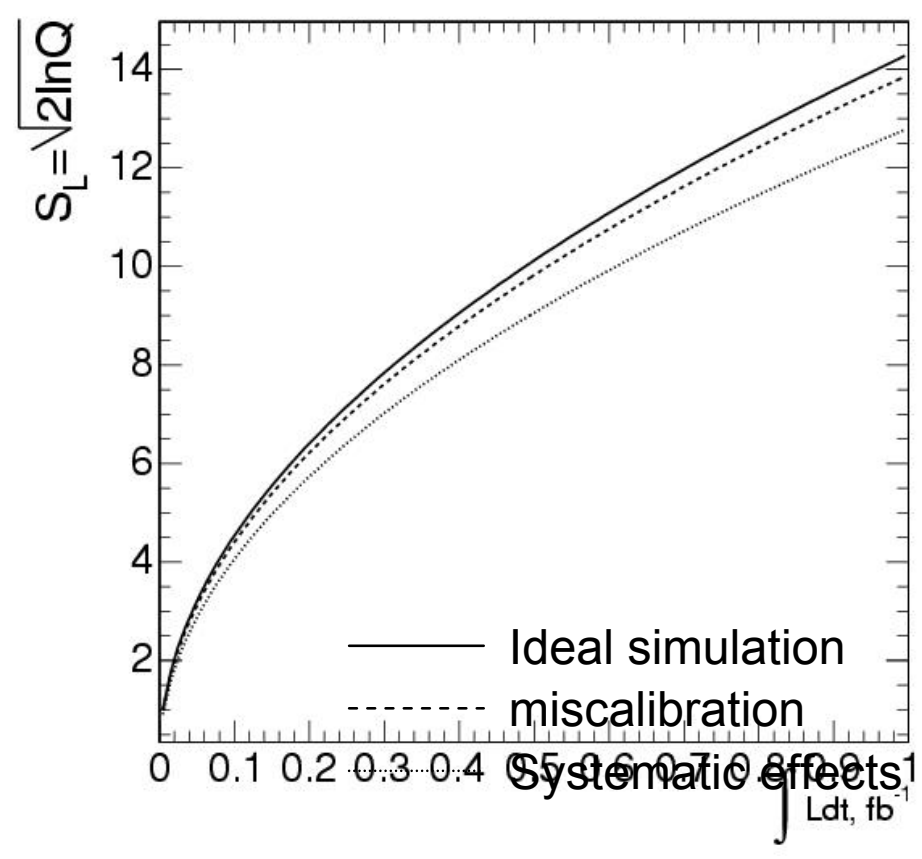

$5 \sigma$ discovery at:

- $\mathrm{ZZ}: \sim 1 \mathrm{fb}^{-1}$

-WZ : -150 $\mathrm{pb}^{-1}$ 


\section{Data for Diboson Studies(ATLAS)}

\begin{tabular}{|c|c|c|c|}
\hline Process & MC data & Process & MC data \\
\hline $\mathrm{ZW}^{+} \rightarrow 2 e / 2 \mu+\mathrm{X}$ & 26033 & ttbar $\rightarrow \ell+x$ & $1.96 \times 10^{5}$ \\
\hline $\mathrm{ZW}^{-} \rightarrow 2 \mathrm{e} / 2 \mu+\mathrm{X}$ & 29085 & $Z(@$ Peak $) \rightarrow$ ee $/ \mu \mu / \tau \tau$ & $2.30 \times 10^{6}$ \\
\hline $\mathrm{ZZ} \rightarrow 4 \mathrm{e}, 4 \mu, 2 \mathrm{e} 2 \mu$ & 19933 & $W \rightarrow e / \mu / t+v$ & $1.61 \times 10^{6}$ \\
\hline$W W \rightarrow e v+x$ & 32056 & $W+$ jets $\rightarrow c+x$ & $1.59 \times 10^{6}$ \\
\hline ZZ(pythia) $\rightarrow 4 \ell(e, \mu)$ & $4.66 \times 10^{4}$ & $Z+$ jet $\rightarrow$ ee $/ \mu \mu / \tau \tau$ & $5.80 \times 10^{6}$ \\
\hline $\mathrm{Zbb} \rightarrow 4 \ell$ & $4.99 \times 10^{4}$ & $D Y Z / \gamma \rightarrow \ell^{+} \ell(e, \mu, \tau)$ & $1.67 \times 10^{7}$ \\
\hline $\bar{Z} \gamma \rightarrow u(e, \mu)$ & $2.50 \times 10^{4}$ & & \\
\hline
\end{tabular}

- Data produced in ATLAS GRID, and Michigan ATLAS computer cluster

- Background (pythia 6.2), $10^{6} \sim 10^{7}$ for Z+jet, W+jet, DY, $W+j e t$ and $W \rightarrow$ lepton

- Signal events produced by MC@NLO (v2.3)-Jimmy, W/Z width effect is not included ( $v 3.1$ has included width) 
WZ
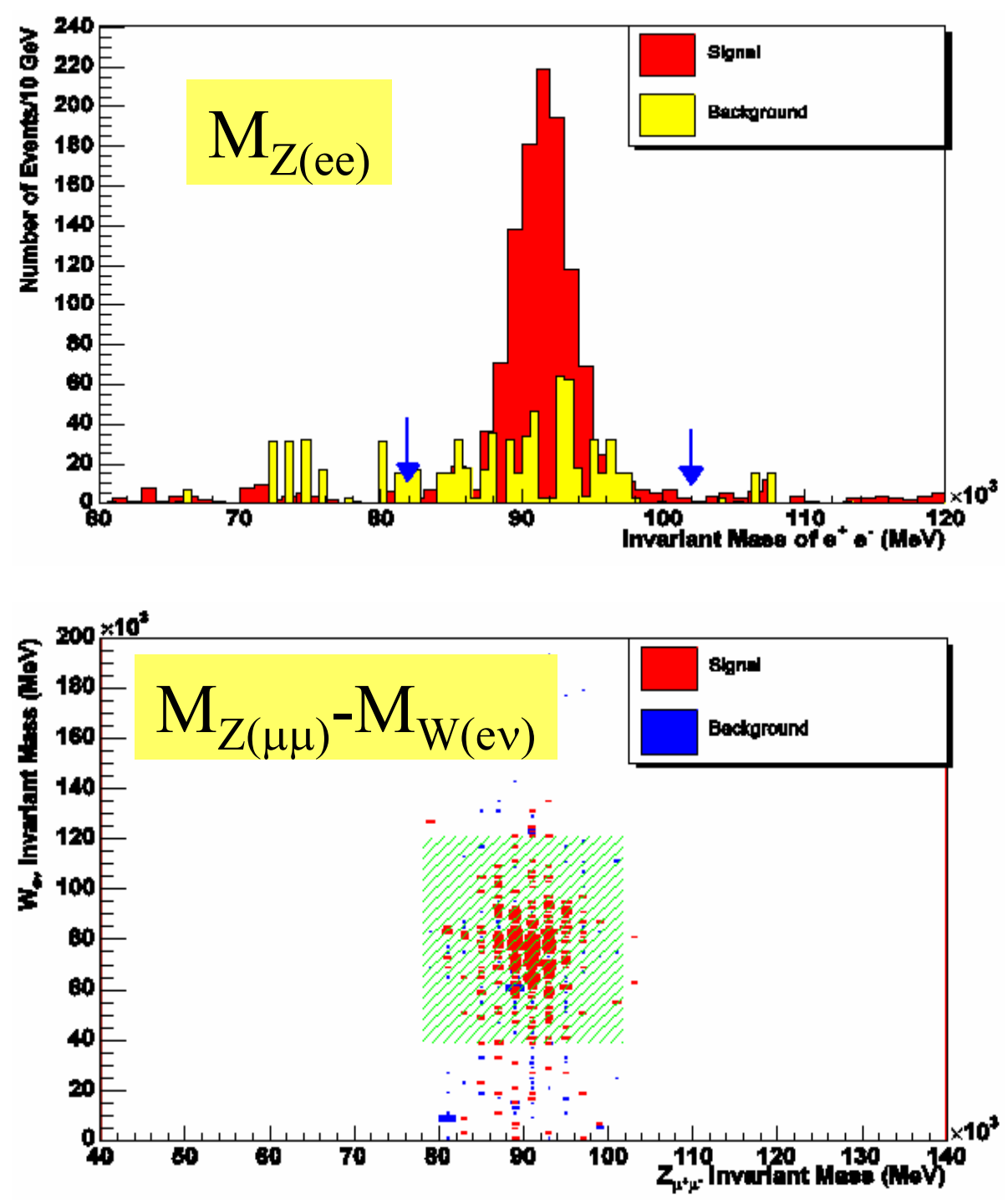

WW
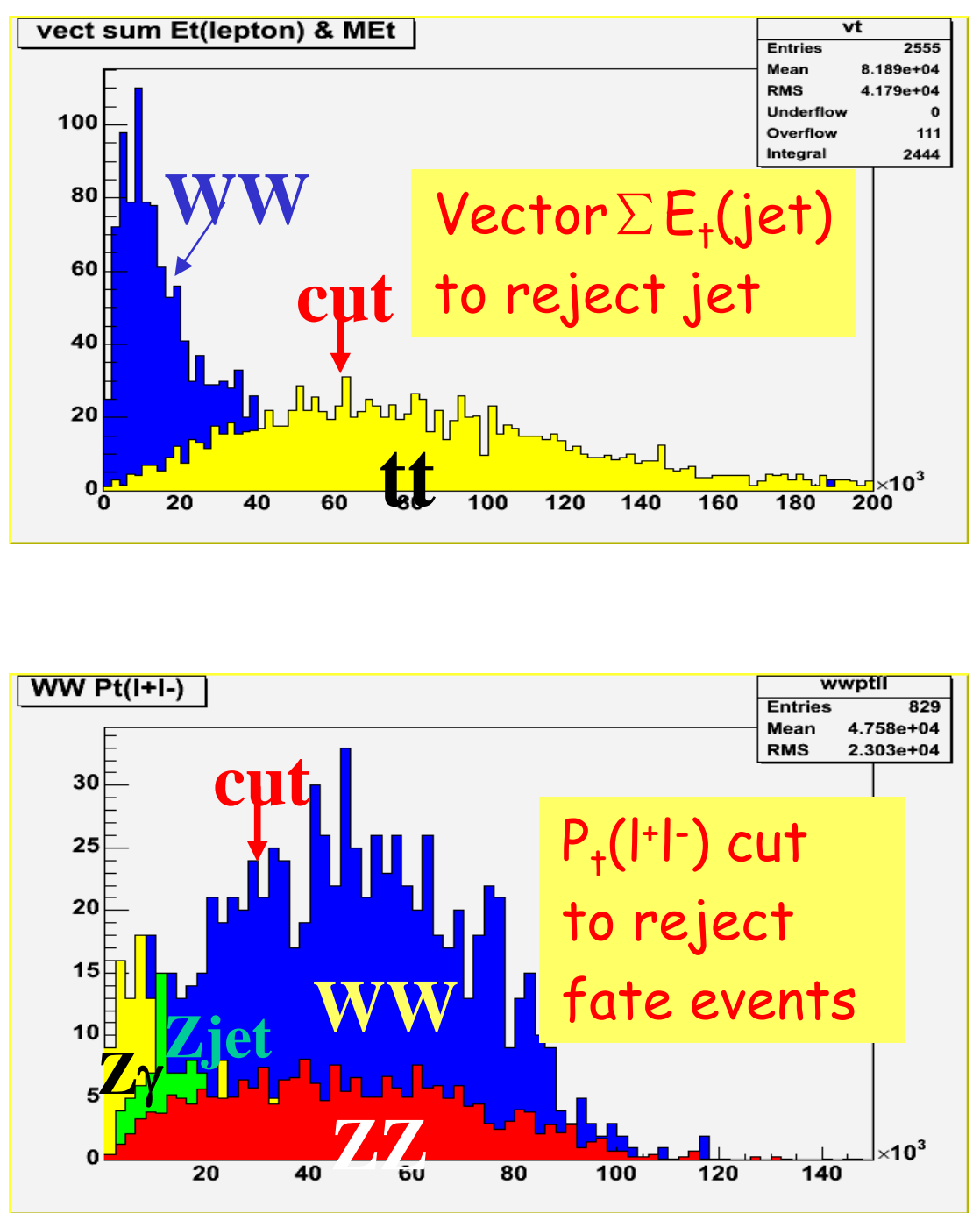


\section{ZW Signal and Backgrounds (ATLAS)}

(for $1 \mathrm{fb}^{-1}$ data)

\begin{tabular}{|c|c|c|c|c|c|}
\hline & $N_{\text {eee }}$ & $N_{\text {ee } \mu}$ & $\mathbf{N}_{\mu \mu e}$ & $\mathbf{N}_{\mu \mu \mu}$ & $N_{\text {total }}\left(1 \mathrm{fb}^{-1}\right)$ \\
\hline$N_{\text {signal }}$ & 16.9 & 17.1 & 21.9 & 19.8 & 75.7 \\
\hline$N_{b k g}$ & 1.71 & 0.88 & 1.73 & 2.00 & 6.32 \\
\hline$S / B$ & 9.84 & 19.4 & 12.7 & 9.92 & 12.0 \\
\hline$S / \sqrt{ } B$ & 12.9 & 18.2 & 16.7 & 14.0 & 30.1 \\
\hline
\end{tabular}

Background Contributions (\%)

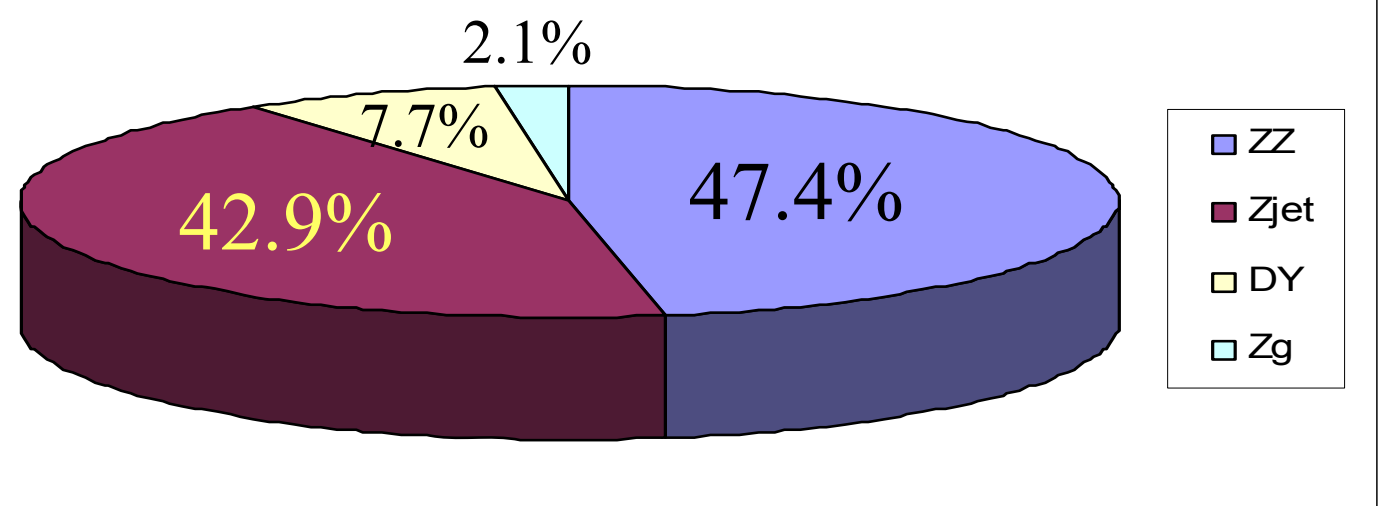




\section{WW Signal and Background (ATLAS)}

(for $1 \mathrm{fb}^{-1}$ data)

\begin{tabular}{|c|c|c|c|c|}
\hline Process & $N_{e e}$ & $N_{\mu \mu}$ & $N_{e \mu}$ & $N_{\text {total }}$ \\
\hline$W W \rightarrow \mid \mathrm{V}+\mathrm{X}(\mathrm{I}=e, \mu)$ & 36.7 & 37.6 & 284.4 & 358.7 \\
\hline Total background & 188.6 & 112.1 & 59.4 & 360.1 \\
\hline S/B & 0.19 & 0.34 & 4.79 & 1.0 \\
\hline S/V $\mathrm{B}$ & 2.67 & 3.55 & 36.9 & 18.9 \\
\hline
\end{tabular}

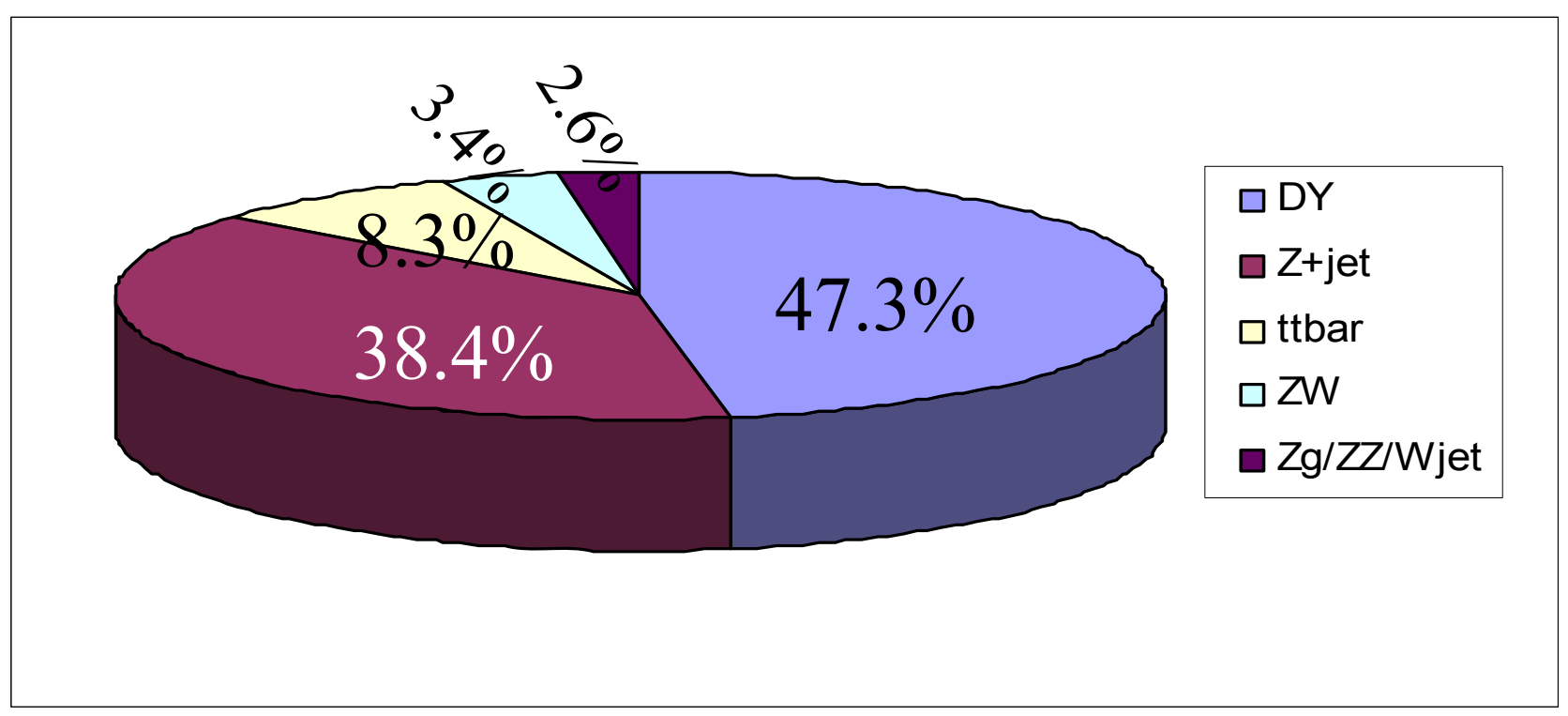




\section{Invariant Mass and Pt Distributions for $\mathrm{ZZ}$}

For $1 \mathrm{fb}^{-1}$ data at ATLAS
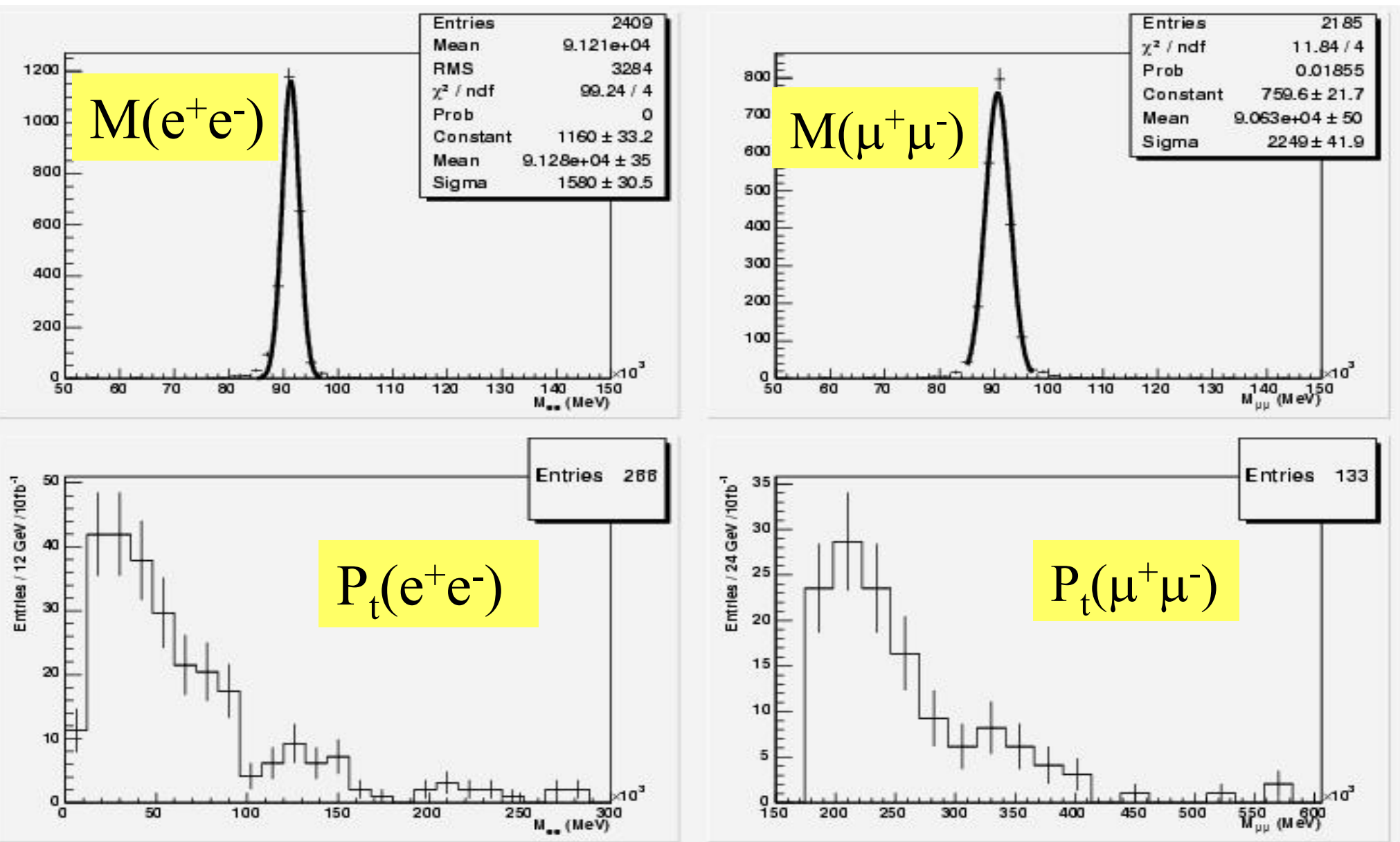

- 13 events candidates

- Background free with current statistics 


\section{Boosted Decision Trees (BDT) and Artificial Neural Network (ANN)}

— signal —background

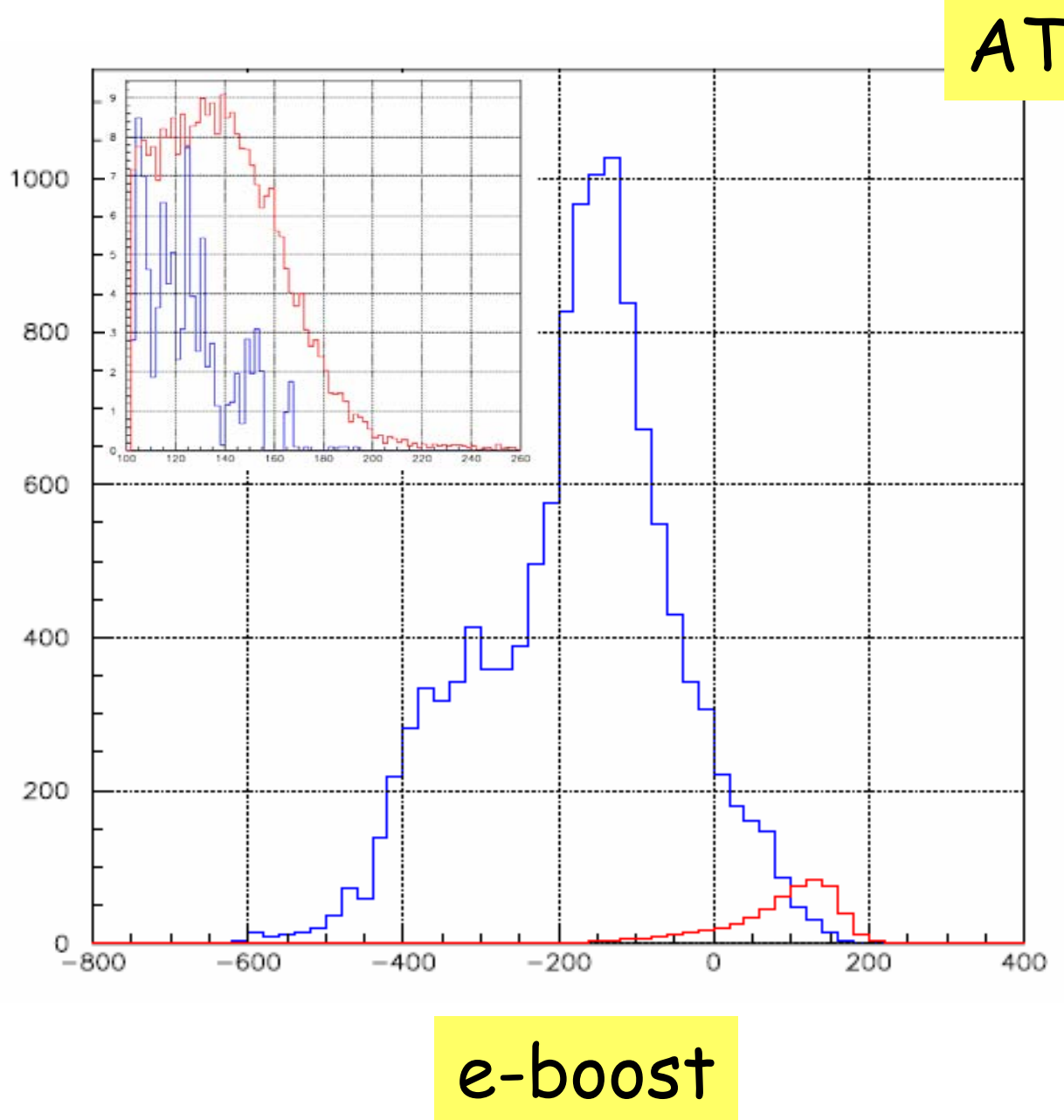

ATLAS

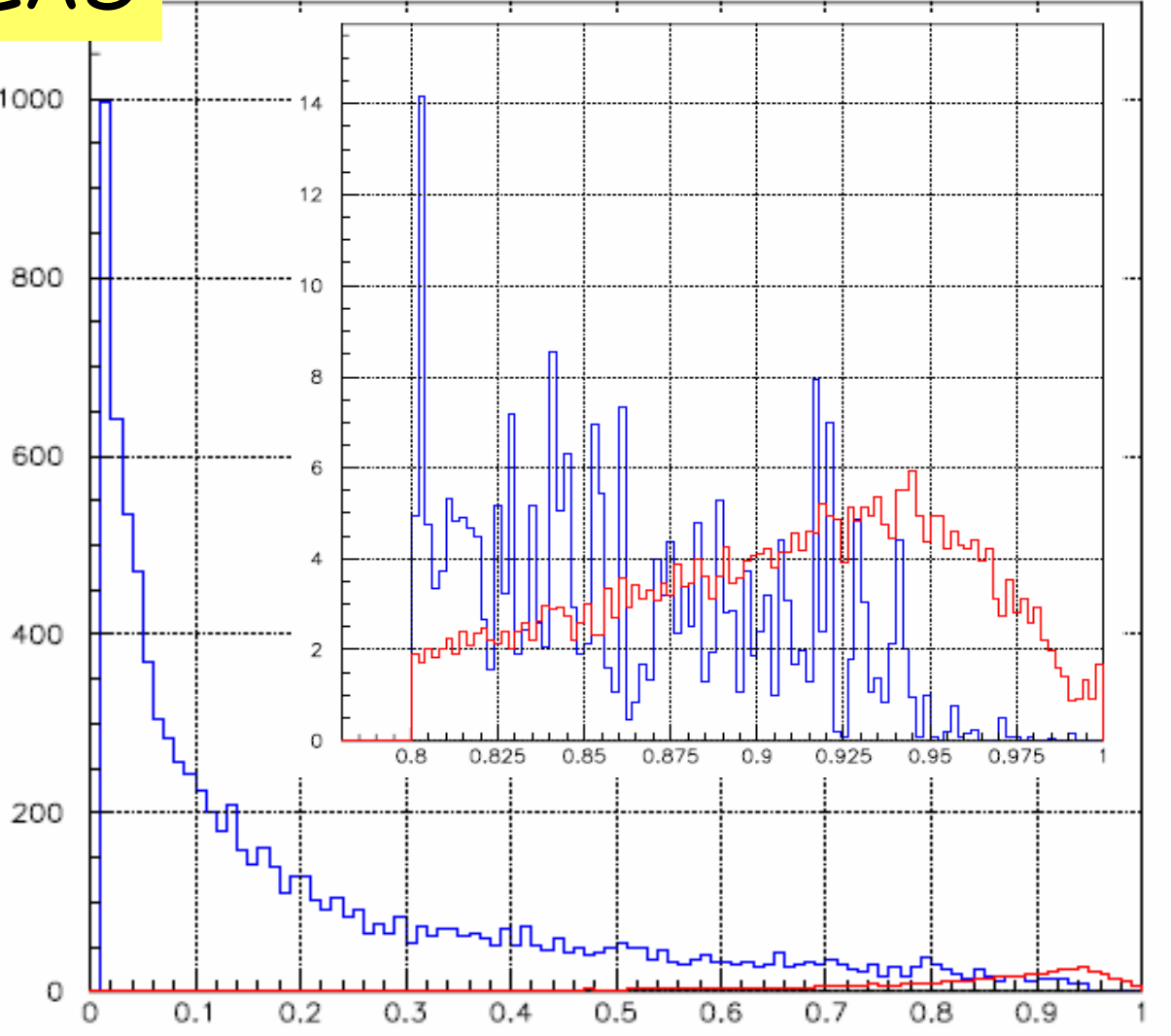

ANN 


\section{Comparison of the efficiencies of BDT/ANN}

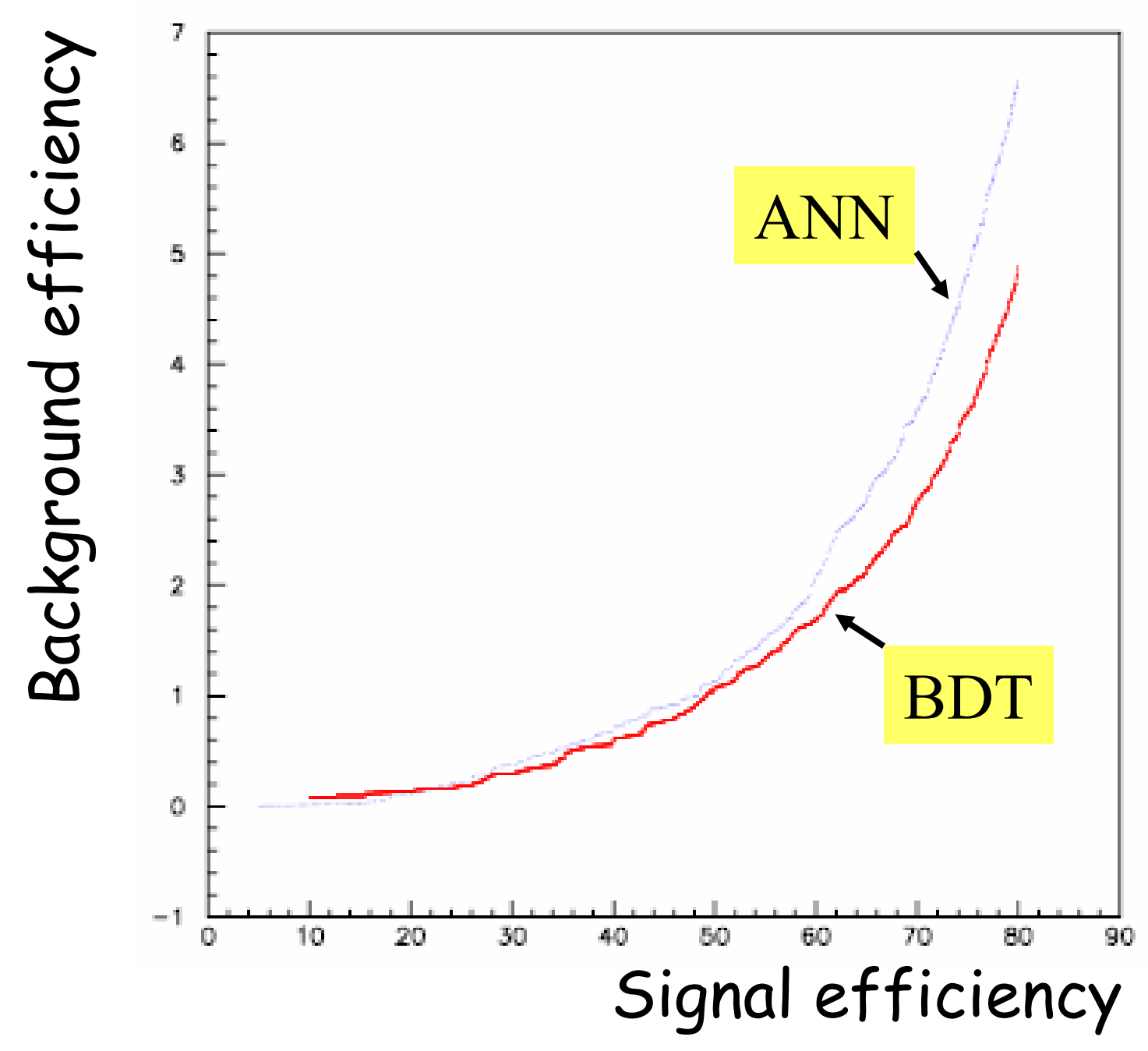




\section{Summary}

- ZZ, WZ and WW signals are expected to be established at CMS and ATLAS with 100 $\mathrm{pb}^{-1} \sim 1 \mathrm{fb}^{-1}$

Expected signal and background with $1 \mathrm{fb}^{-1}$ data at CMS/ATLAS

\begin{tabular}{|c|c|c|}
\hline & $\begin{array}{c}C M S \\
\left(N_{s} / N_{b}\right)\end{array}$ & $\begin{array}{c}\text { ATLAS } \\
\left(N_{s} / N_{b}\right)\end{array}$ \\
\hline$W W$ & $\times$ & $284.4 / 59.4(e \mu)$ \\
\hline$Z Z$ & $7.1 / 0.4(4 e)$ & $13 / 0(4 e, 4 \mu, 2 e 2 \mu)$ \\
\hline$W Z(31,1=e, \mu)$ & $97 / 23$ & $75.7 / 6.3$ \\
\hline
\end{tabular}

- Anonymous gauge boson coupling can be probed with a few $\mathrm{fb}^{-1}$ data

- To improve the TGC's with LHC data, it's crucial to build the TGCs into MC@NLO event generator 


\section{Backup Slides}


Muon spectrometer

$\cdot \sigma / \mathrm{p}_{\mathrm{T}} \sim 2-7 \%$

• $|\eta|<2.7,|\eta|<2.5$ ( precision phys.)

\section{EM Calorimetry}

- $\sigma / \mathrm{E} \sim 10 \% / \sqrt{\mathrm{E}}(\mathrm{GeV}) \oplus 1 \%$

- $|\eta|<3.2,|\eta|<2.5$ (fine granularity)

Forward Calorimeters
Length: $\sim 46 \mathrm{~m}$

Radius : $12 \mathrm{~m}$

Weight : 7000 tons Channels: $\sim 10^{8}$ $\mathrm{L}_{\text {cable }}$ : $3000 \mathrm{~km}$
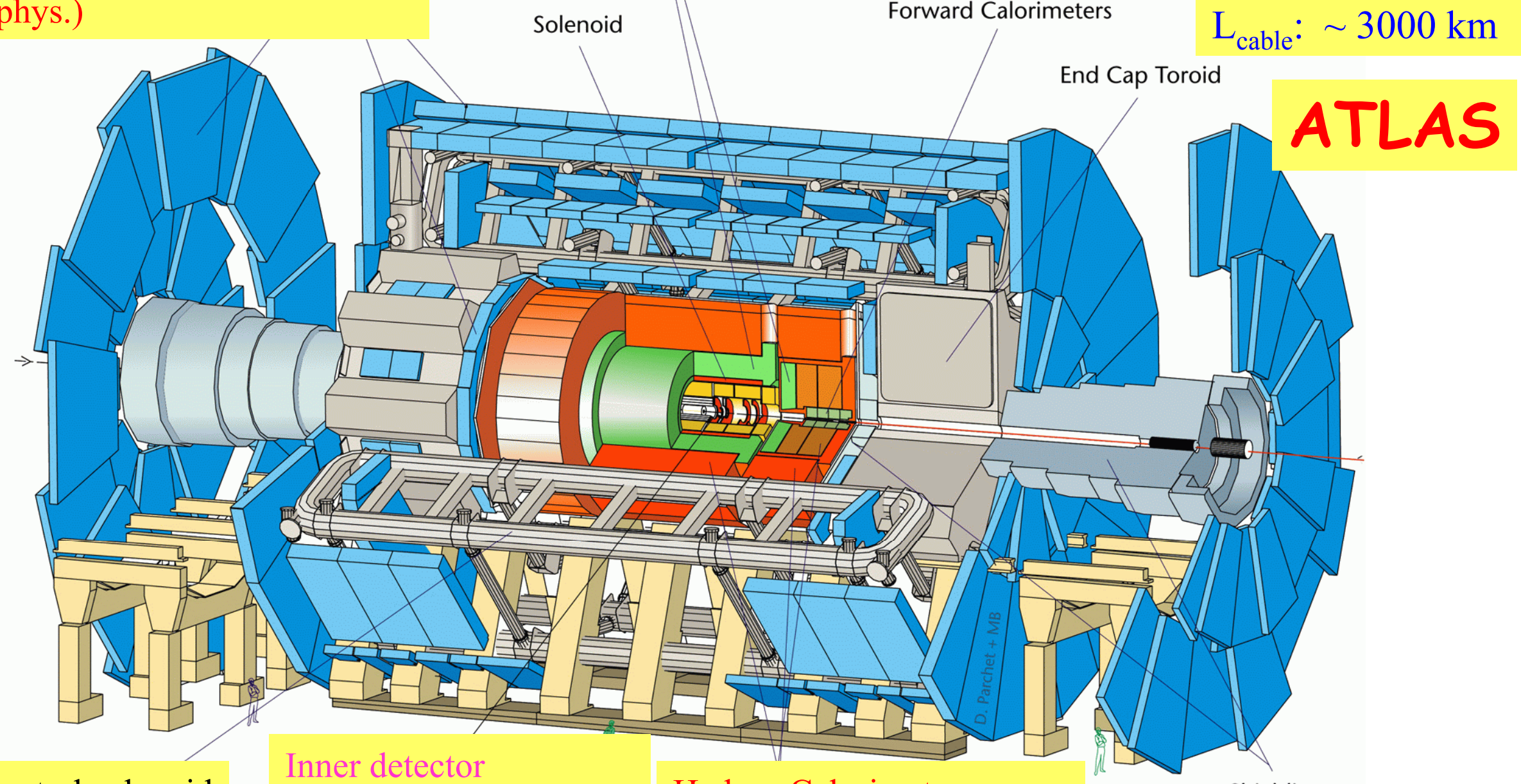

Central solenoid

- $2 \mathrm{~T}$

Inner detector

- $\sigma / \mathrm{p}_{\mathrm{T}} \sim 0.05 \% \mathrm{p}_{\mathrm{T}}(\mathrm{GeV})$ $\oplus 0.1 \%$;

$\bullet|\eta|<2.5$
Hadron Calorimeter

Shielding
- $\sigma / \mathrm{E} \sim 50 \% / \sqrt{\mathrm{E}}(\mathrm{GeV}) \oplus 3 \%$

$\cdot|\eta|<3$ 
EM calorimeter:

- Lead tungstate

- $\sigma / \mathrm{E}=5 \% / \sqrt{\mathrm{E}}(\mathrm{GeV}) \oplus 2 \%$
Magnet solenoid

- $4 \mathrm{~T}$
Muon spectrometer

- $\mathrm{DT}+\mathrm{CSA}+\mathrm{RPC}$

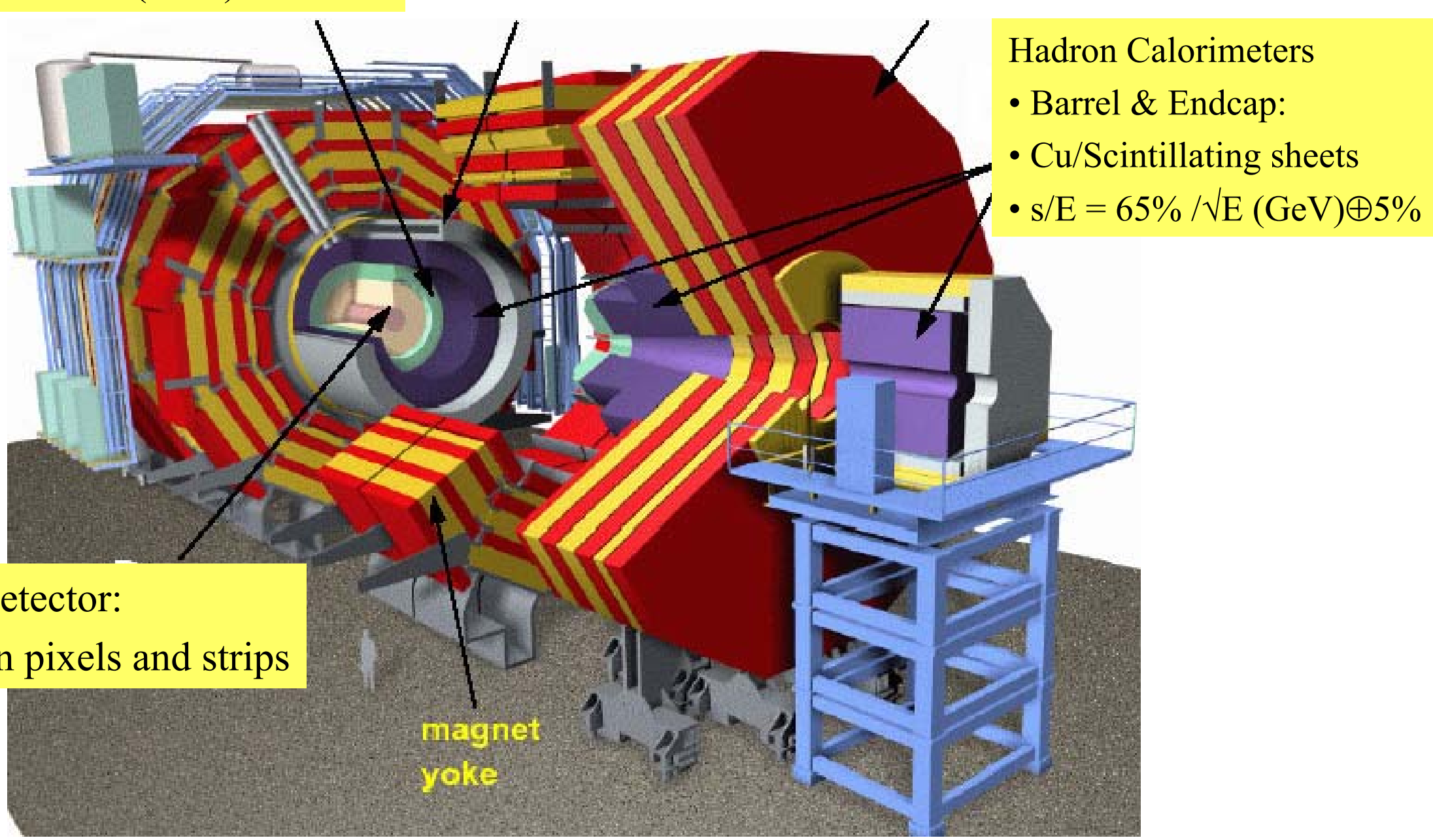

Inner Detector:

- Silicon pixels and strips 


\section{Triple Gauge Boson Couplings}

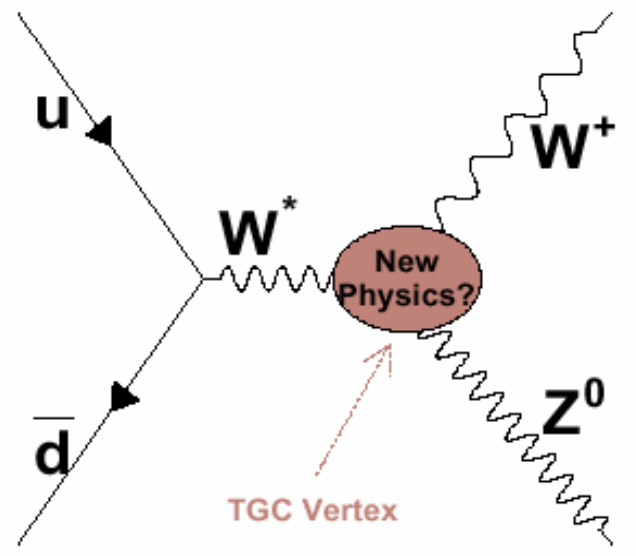

non-abelian $S U(2) L \times U(1)$ y gauge group (foundation of SM!)

Open window to electroweak symmetry breaking mechanism

LHC: orders of magnitude Improvement over LEP/Tevatron
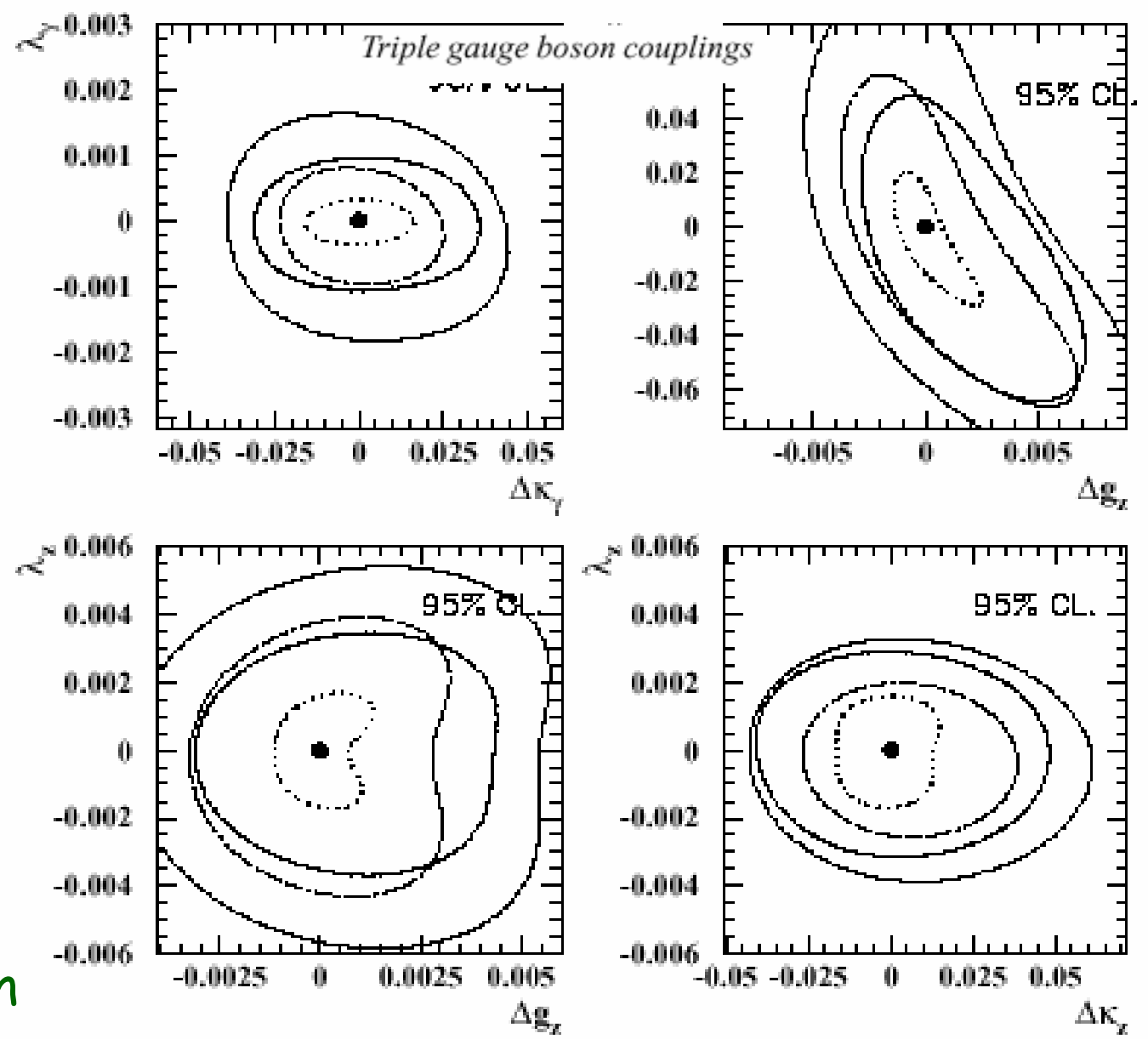

Expected $95 \%$ C.L. constrains contours (outer-> inside): $\left(14 \mathrm{TeV}, 100 \mathrm{fb}^{-1}\right),\left(28 \mathrm{TeV}, 100 \mathrm{fb}^{-1}\right),\left(14 \mathrm{TeV}, 1000 \mathrm{fb}^{-1}\right),\left(28 \mathrm{TeV}, 1000 \mathrm{fb}^{-1}\right)$ 


\section{Quartic Gauge Boson Couplings}

$100 \mathrm{fb}^{-1}$

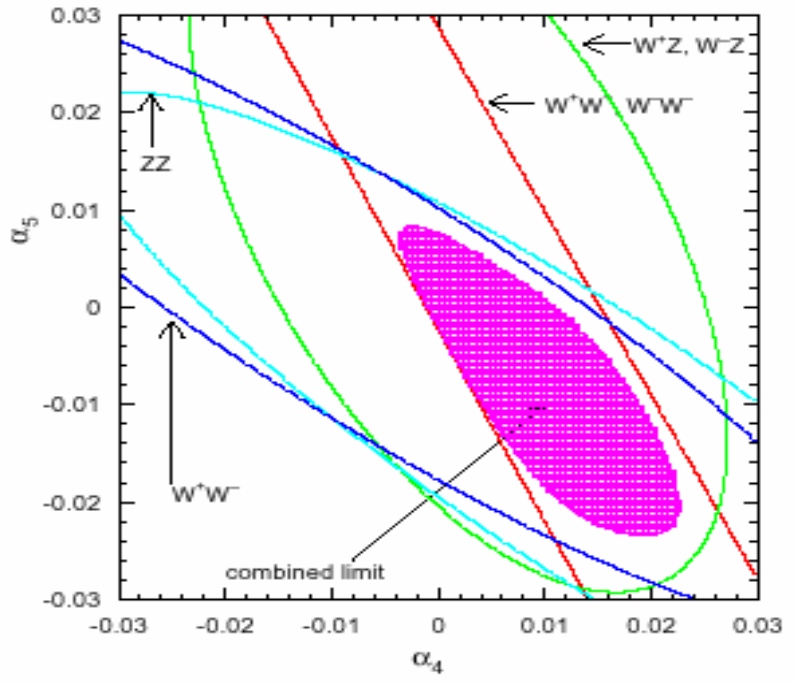

$100 \mathrm{fb}^{-1}$

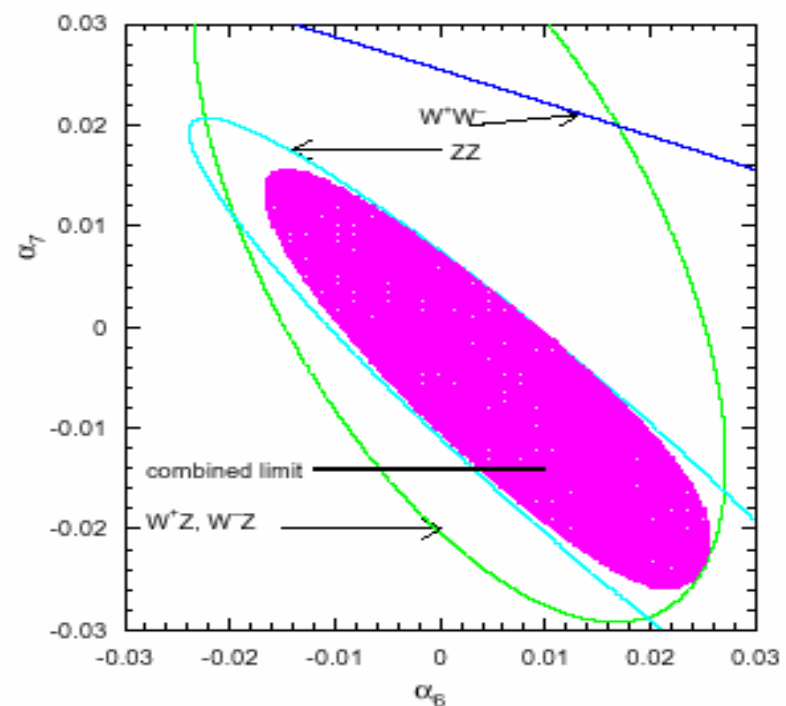

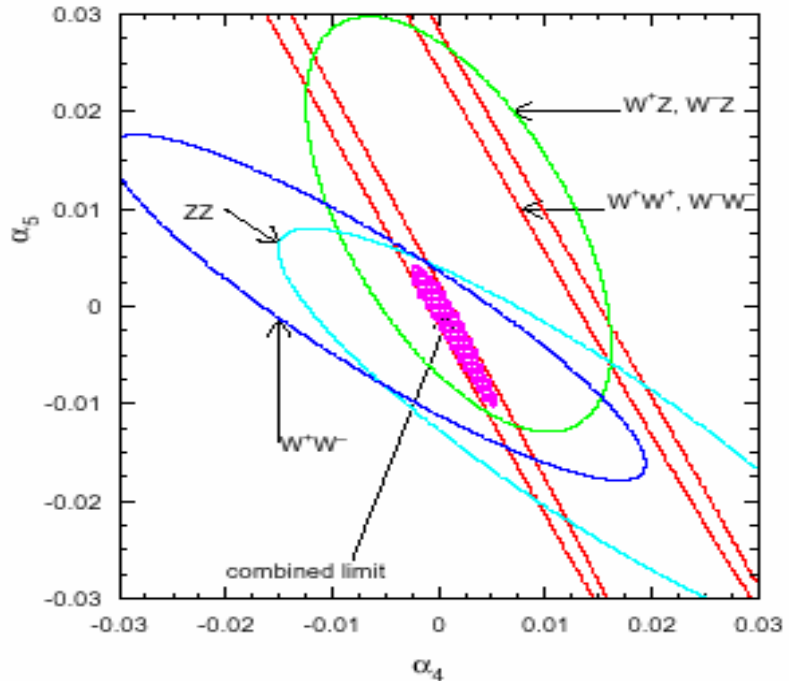

$6000 \mathrm{fb}^{-1}$

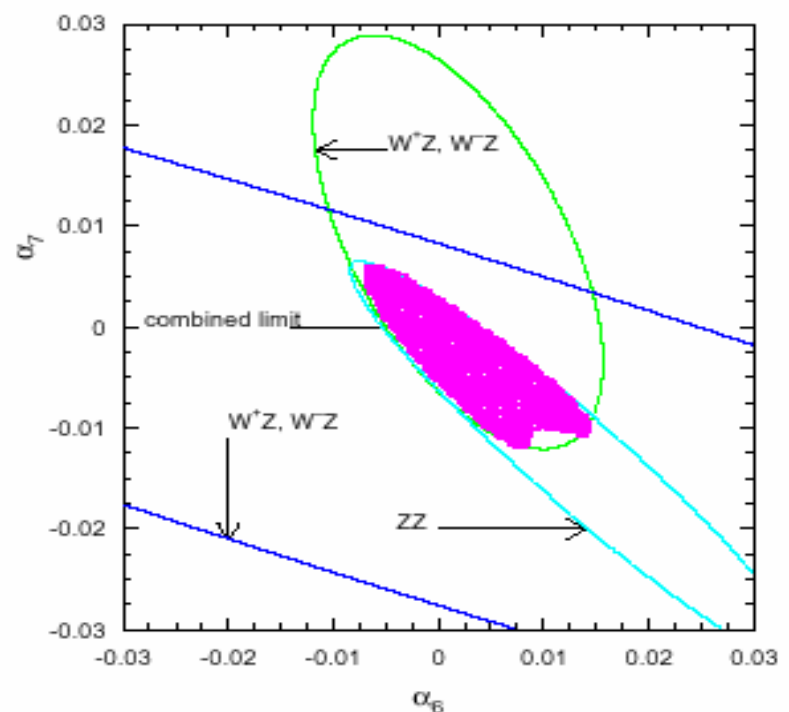

$6000 \mathrm{fb}^{-1}$ 


\section{Systematic Uncertainties (CMS)}

WZ

\begin{tabular}{lcc}
\hline \hline Systematic source & Cross section & Significance \\
\hline Luminosity & 10.0 & - \\
Trigger efficiency & 1.0 & 1.0 \\
Electron identification & 2.6 & 5.2 \\
Muon identification & 3.4 & 6.8 \\
Jet energy scale & 5.0 & 5.0 \\
$Z^{0} b \bar{b}$ subtraction & 12.0 & 12.0 \\
$Z^{0} Z^{0} \rightarrow 4 l$ subtraction & 4.0 & 4.0 \\
PDF uncertainty & - & 3.5 \\
\hline Total & 17.4 & 20.8 \\
\hline \hline
\end{tabular}

ZZ

Systematic uncertainties on cross section

\begin{tabular}{lll}
\hline \hline Source of systematic uncertainty & $\int L d t=1 \mathrm{fb}^{-1}$ & $\int L d t=10 \mathrm{fb}^{-1}$ \\
\hline Luminosity & 10.0 & 5.0 \\
Trigger efficiency & 1.0 & 1.0 \\
Background subtraction & 0.6 & 0.6 \\
$Z^{0} \gamma^{*}$ subtraction & 1.2 & 1.2 \\
Electron identification & $4 \times 2.0$ & $4 \times 1.5$ \\
\hline Total & 12.9 & 7.9 \\
\hline \hline
\end{tabular}

Systematic uncertainties on significance

\begin{tabular}{lll}
\hline \hline Source & $\int L d t=1 \mathrm{fb}^{-1}$ & $\int L d t=10 \mathrm{fb}^{-1}$ \\
\hline Trigger efficiency & 1.0 & 1.0 \\
Background subtraction & 0.6 & 0.6 \\
$Z^{0} \gamma^{*}$ subtraction & 1.2 & 1.2 \\
Electron identification & $4 \times 2.0$ & $4 \times 1.5$ \\
PDF and QCD scale factor & 6.4 & 6.4 \\
\hline Total & 18.4 & 14.9 \\
\hline \hline
\end{tabular}

